\title{
On Strict Homogeneous Lyapunov Function for Generalized Homogeneous PI Controller
}

\author{
Yu Zhou, Andrey Polyakov, Gang Zheng
}

\begin{abstract}
This paper investigates a generalized homogeneous PI (Proportional-Integral) control for a linear plant. A Lyapunov function for analysis of the closed-loop system is designed. For negative homogeneity degree, the obtained Lyapunov function becomes a strict Lyapunov function allowing an advanced analysis to be provided. In particular, a class of the disturbances to be rejected by the control law is characterized, a maximum control magnitude and the settling-time of the closedloop system are estimated.
\end{abstract}

\section{INTRODUCTION}

The integral term in a linear controller is introduced to reject non-vanishing perturbations. PI controllers have been applied in various fields due to the relative simplicity and robustness, in addition to satisfactory performance. The PI controller design in frequency domain is well-developed for SISO (single input single output) linear system. For the MIMO (multiple-input and multiple-output) linear system, the PI parameters can be tuned using LMIs. The integral term also works for nonlinear control design [24], [19], [7], [11], [12]. As known, a memoryless discontinuous controller (e.g. sliding mode algorithm) can also stabilize a system with non-vanishing perturbations. Theoretically compared to continuous integral control, sliding mode control [25] can reject a lager class of bounded perturbations with better robustness properties. But the main problem of a discontinuous controller in practice is "chattering", introducing the integral term may potentially reduce the "chattering" caused by the discontinuity. A classical example of PI integral sliding mode controller is the super-twisting algorithm [14].

Homogeneity is a dilation symmetry. All linear and a lot of essentially nonlinear models of mathematical physics are homogeneous (symmetric) in a generalized sense [21]. Homogeneity is widely used in control theory for system analysis, regulation and observer design ([9], [1], [15], [18], [3], [2], [21]). Homogeneity has some useful features such as: local stability means global one; the rate of convergence of homogeneous system is defined by its homogeneity degree; homogeneous systems are robust with respect to external perturbations and time delays.

Implicit Lyapunov function (ILF) combines two important notions from mathematical and stability analysis: Implicit

This work is partially supported by Chinese Science Council (CSC), the National Natural Science Foundation of China under Grant 62050410352 and the Ministry of Science and Higher Education of Russian Federation, passport of goszadanie no. 2019-0898

Y. Zhou, A. Polyakov and G. Zheng are with Univ. Lille, Inria, CNRS, Centale Lille, France, e-mail: yu.zhou(andrey.polyakov, gang.zheng)@inria.fr. A. Polyakov is also with ITMO University, SaintPetersburg, Russia function and Lyapunov function. In [28], the design of a homogeneous controller using ILF shows that the ILF makes the gain tune can be reduced to an LMI problem and systematized the control design and analysis.

A weighted homogeneous PI algorithm is designed in [16]. The linear geometric homogeneous PI controller was considered in [21] and utilized for an upgrade of existing linear control law to a generalized homogeneous one. In [27] on real experiments it was shown that such an upgrade may essentially improve the control performance. However, the theoretical analysis of this very efficient control law is not complete. Inspired by [17] we introduce a strict Lyapunov function for the closed-loop system with such a generalized homogeneous PI algorithm. This function allows us to present the missed analysis for the case of negative homogeneity degree.

- We provide an estimate of the magnitude of the homogeneous PI controller under consideration.

- We derive an estimate the settling time of the closedloop system controlled by the homogeneous PI algorithm with negative degree.

- We characterize a class of perturbations to be rejected by means of the homogeneous PI algorithm.

The main problem of integral control is the saturation since the control signal in integral control is obtained through time integration. Estimating the magnitude and settling time helps solve the wind-up problem and actuator constrained situation. A class of perturbations, that the homogeneous PI controller can reject, shows that the homogeneous PI controller has better robustness properties than the proportional algorithm.

The paper is organized as follows. Section II presents the basic knowledge of the homogeneous systems and the canonical homogeneous norm. Section III recalls the form of homogeneous proportional control. Section IV introduces the homogeneous proportional-integral control and analyzes it via a strictly Lyapunov function. In section $\mathrm{V}$, a simulation of linear MIMO system is used to validate the theorem.

Notation. $\mathbb{R}$ is the set of real numbers, $\mathbb{R}_{+}=\{x \in \mathbb{R}$ : $x \geq 0\} ;\|\cdot\|$ is a norm in $\mathbb{R}^{n} ; \mathbf{0}$ denotes the zero vector from $\mathbb{R}^{n} ; \operatorname{diag}\left\{\sigma_{i}\right\}_{i=1}^{n}$ is the diagonal matrix with elements $\sigma_{i} ; P \succ 0(\prec 0, \succeq 0, \preceq 0)$ for $P \in \mathbb{R}^{n \times n}$ means that the matrix $P$ is symmetric and positive (negative) definite (semidefinite); $C(X, Y)$ denotes the space of continuous functions $X \rightarrow Y$, where $X, Y$ are subsets of normed vector spaces; $C^{p}(X, Y)$ is the space of functions continuously differentiable at least up to the order $p ; \lambda_{\min }(P)$ and $\lambda_{\max }(P)$ represent the minimal and maximal eigenvalue of a matrix 
$P=P^{\top}$; for $P \succeq 0$ the square root of $P$ is a matrix $M=P^{\frac{1}{2}}$ such that $M^{2}=P$.

\section{PROBlEM STATEMENT}

In this paper, we consider a linear system with pertubations as:

$$
\dot{x}=A x+B u+g(t, x)
$$

where $x \in \mathbb{R}^{n}$ is the state vector, $u \in \mathbb{R}^{m}$ is the control input and $g(t, x)$ is the generalized pertubation. The pair of $\{A, B\}$ is controllable.

A memoryless continuous state feedback control can stabilize the system (1) when $g(t, x)$ is a vanishing perturbation. However, it cannot stabilize the system when $g(t, x)$ is aconstant or other non-vanishing perturbation. Hence, we aim to design an integral control to reject a class of matched non-vanishing perturbations. The goal of this paper is to design a generalized homogeneous PI controller to stabilize the system (1) with a finite or nearly fixed-time convergence rate by using a strict Lyapunov function.

\section{Homogeneous Systems}

\section{A. Linear dilations in $\mathbb{R}^{n}$}

By definition, the homogeneity is a dilation symmetry [29], [10], [9], [4], [21]. A dilation [6] is a one-parameter group $\mathbf{d}(s), s \in \mathbb{R}$ of transformations satisfying the limit property $\lim _{s \rightarrow s^{\infty}}\|\mathbf{d}(s) x\|=e^{s^{\infty}}, s^{\infty}= \pm \infty, \forall x \neq \mathbf{0}$.

Examples of dilations in $\mathbb{R}^{n}$ are

- Uniform dilation (L. Euler, 18th century): $\mathbf{d}(s)=e^{s} I$, where $I$ is the identity matrix $\mathbb{R}^{n}$;

- Weighted dilation (Zubov 1958) : $\mathbf{d}(s)=$ $\left(\begin{array}{ccc}e^{r_{1}}{ }^{s} & \ldots & 0 \\ \cdots & \cdots & \ldots \\ 0 & \cdots & e^{\ddot{r_{n}}}\end{array}\right)$, where $r_{i}>0, i=1,2, \ldots, n$.

- Geometric dilation is a flow generated by unstable $C^{1}$ vector field in $\mathbb{R}^{n}$ (see [10], [8]).

In this paper we deal only with the so-called linear (geometric) dilation in $\mathbb{R}^{n}$ is defined as follows

$$
\mathbf{d}(s)=e^{s G_{\mathbf{d}}}:=\sum_{i=0}^{+\infty} \frac{s^{i} G_{\mathbf{d}}^{i}}{i !}, \quad s \in \mathbb{R}^{n},
$$

where $G_{\mathbf{d}} \in \mathbb{R}^{n \times n}$ is an anti-Hurwitz matrix called the generator of the dilation $\mathbf{d}$.

Definition 1: A dilation $\mathbf{d}$ is monotone if $s \rightarrow\|\mathbf{d}(s) x\|$ is a monotone increasing function for any $x \neq \mathbf{0}$.

It is worth noting that monotonicity of the dilation may depend of the norm in $\mathbb{R}^{n}$. Also, any linear dilation in $\mathbb{R}^{n}$ is monotone [20] provided that the norm in $\mathbb{R}^{n}$ is defined as follows

$$
\|x\|=\sqrt{x^{\top} P x}, \quad P G_{\mathbf{d}}+G_{\mathbf{d}}^{\top} P \succ 0, P \succ 0 .
$$

\section{B. Canonical Homogeneous Norm}

The linear dilation introduces an alternative norm topology in $\mathbb{R}^{n}$ by means of a homogeneous norm (see, e.g., [5] for an example of a homogeneous norm induced by the weighted dilation).
Definition 2: [20] The functional $\|\cdot\|_{\mathbf{d}}: \mathbb{R}^{n} \rightarrow[0,+\infty)$ defined as $\|\mathbf{0}\|_{\mathbf{d}}=0$ and

$$
\|u\|_{\mathrm{d}}=e^{s_{u}}, \text { where } s_{u} \in \mathbb{R}:\left\|\mathbf{d}\left(-s_{u}\right) u\right\|=1,
$$

is called the canonical homogeneous norm in $\mathbb{R}^{n}$, where $\mathbf{d}$ is a monotone dilation in $\mathbb{R}^{n}$.

For any linear monotone dilation in $\mathbb{R}^{n}$, the canonical homogeneous norm is continuous on $\mathbb{R}^{n}$ and locally Lipschitz continuous on $\mathbb{R}^{n} \backslash\{\mathbf{0}\}$. Moreover, it is differentiable on $\mathbb{R}^{n} \backslash\{\mathbf{0}\}$ provided that $\|\cdot\|$ is given by (3) (see, [20]):

$$
\frac{\partial\|x\|_{\mathbf{d}}}{\partial x}=\|x\|_{\mathbf{d}} \frac{x^{\top} \mathbf{d}^{\top}\left(-\ln \mid x \|_{\mathbf{d}}\right) P}{x^{\top} \mathbf{d}^{\top}\left(-\ln \mid x \|_{\mathbf{d}}\right) P G_{\mathbf{d}} \mathbf{d}\left(-\ln \mid x \|_{\mathbf{d}}\right) x} .
$$

\section{Homogeneous Systems}

Definition 3: A vector field $f: \mathbb{R}^{n} \rightarrow \mathbb{R}^{n}$ (resp. a function $h: \mathbb{R}^{n} \rightarrow \mathbb{R}$ ) is said to be d-homogeneous of degree $\mu \in \mathbb{R}$ if

$$
\begin{gathered}
f(\mathbf{d}(s))=e^{\mu s} \mathbf{d}(s) f(x), \quad \forall x \in \mathbb{R}^{n}, \quad \forall s \in \mathbb{R}, \\
\left(\operatorname{resp} . h(\mathbf{d}(s))=e^{\mu s} h(x), \quad \forall x \in \mathbb{R}^{n}, \quad \forall s \in \mathbb{R}\right),
\end{gathered}
$$

where $\mathbf{d}$ is a linear dilation in $\mathbb{R}^{n}$.

In [20] it is shown that any $\mathbf{d}$-homogeneous system

$$
\dot{x}=f(x), \quad t>0, \quad x(0)=x_{0} \in \mathbb{R}^{n}
$$

is diffeomorphic on $\mathbb{R}^{n} \backslash\{\mathbf{0}\}$ to a standard homogeneous system. This means that many important results known for standard and weighted homogeneous systems hold for linear homogeneous systems as well. Following theorem is the straightforward corollary of Zubov-Rosier theorem on homogeneous Lyapunov function [29], [23].

Theorem 1: Let $f$ be a continuous d-homogeneous vector field of degree $\mu \in \mathbb{R}$. The system (5) is globally asymptotically stable if and only if there exists a positive definite d-homogeneous function $V: \mathbb{R}^{n} \rightarrow[0,+\infty)$ such that $V \in C\left(\mathbb{R}^{n}\right) \cap C^{1}\left(\mathbb{R}^{n} \backslash\{\mathbf{0}\}\right)$ and

$$
\dot{V}(x) \leq-\rho V^{1+\mu}(x), \quad \forall x \neq 0 .
$$

The latter theorem immediately implies that asymptotically stable homogeneous system (5) is

- globally uniformly finite-time stable ${ }^{1}$ for $\mu<0$, and $T\left(x_{0}\right) \leq \frac{1}{-\rho \mu} V_{0}^{-\mu}$, where $V_{0}=V\left(x_{0}\right) ;$

- globally uniformly exponentially stable for $\mu=0$;

- globally uniformly nearly fixed-time stable $^{2}$ for $\mu>0$, $T_{\max }=\frac{1}{\rho \mu r^{\mu}}$.

\section{Proportional Homogeneous Control for Linear Plants}

Let us consider the special linear control system of (1) as

$$
\dot{x}=A x+B u(x)+g(t, x), \quad t>0, \quad x(0)=x_{0}
$$

The disturbance-free (i.e., $g=0$ ) system (6) is $\mathbf{d -}$ homogeneously stabilizable with a degree $\mu \neq 0$ if and only if the pair $\{A, B\}$ is controllable [28].

\footnotetext{
${ }^{1}$ The system (5) is finite-time stable if it is Lyapunov stable and $\exists T\left(x_{0}\right)$ : $\|x(t)\|=0, \forall t \geq T\left(x_{0}\right), \forall x_{0} \in \mathbb{R}^{n}$.

${ }^{2}$ The system (5) is uniformly nearly fixed-time stable it is Lyapunov stable and $\forall r>0, \exists T_{r}>0:\|x(t)\|<r, \forall t \geq T_{r}$ independently of $x_{0} \in \mathbb{R}^{n}$.
} 
Theorem 2: [28] If the linear equation

$$
A G_{0}+B Y_{0}=G_{0} A+A, \quad G_{0} B=\mathbf{0} .
$$

has a solution $G_{0} \in \mathbb{R}^{n \times n}$ and $Y_{0} \in \mathbb{R}^{m \times n}$ such that $G_{0}-I_{n}$ is invertible, then for any $\mu \neq 0$ such that $G_{\mathbf{d}}=I_{n}+\mu G_{0}$ is anti-Hurwitz, the disturbance-free system (6) can always be homogeneously stabilized by the following control

$$
u(x)=K_{0} x+\|x\|_{\mathbf{d}}^{1+\mu} K \mathbf{d}\left(-\ln \|x\|_{\mathbf{d}}\right) x, \quad K=Y X^{-1}
$$

with any $\rho>0, \mu \geq-1, K_{0}=Y_{0}\left(G_{0}-I_{n}\right)^{-1}$ any $X \in$ $\mathbb{R}^{n \times n}, Y \in \mathbb{R}^{m \times n}$ by solving the following algebraic system

$$
\left\{\begin{array}{l}
X A_{0}^{\top}+A_{0} X+Y^{\top} B^{\top}+B Y+\rho\left(X G_{\mathbf{d}}^{\top}+G_{\mathbf{d}} X\right)=\mathbf{0} \\
X G_{\mathbf{d}}^{\top}+G_{\mathbf{d}} X \succ 0, \quad X \succ 0
\end{array}\right.
$$

$A_{0}=A+B K_{0}$, where the canonical homogeneous norm $\|\cdot\|_{\mathbf{d}}$ is induced by the norm $\|x\|=\sqrt{x^{\top} X^{-1} x}$. Moreover, if

$$
2 \frac{g^{\top}(t, x) \mathbf{d}^{\top} X^{-1} \mathbf{d}\left(-\ln \|x\|_{\mathbf{d}}\right) x}{x^{\top} \mathbf{d}^{\top}\left(-\ln \|x\|_{\mathbf{d}}\right) X^{-1} G_{\mathbf{d}} \mathbf{d}\left(-\ln \|x\|_{\mathbf{d}}\right) x}<\rho\|x\|_{\mathbf{d}}^{\mu}, \quad \forall t \geq 0,
$$

then the perturbed system (6) is

- globally uniformly finite-time stable for $\mu<0$;

- globally uniformly exponentially stable for $\mu=0$;

- globally nearly fixed-time stable for $\mu>0$.

The proof of this theorem is based on the use of the canonical homogeneous norm as a Lyapunov function of the closedloop system (6), (8) with $g=\mathbf{0}$ :

$$
\frac{d}{d t}\|x(t)\|_{\mathbf{d}}=-\rho\|x(t)\|_{\mathbf{d}}^{1+\mu}
$$

The disturbance $g$ rejected by the control law is characterized by the inequality (10), which is obtained from (4) and (11) using

$$
\begin{aligned}
\frac{d\|x(t)\|_{\mathbf{d}}}{d t}= & -\rho\|x(t)\|_{\mathbf{d}}^{1+\mu} \\
& +2\|x\|_{\mathbf{d}} \frac{g^{\top}(t, x) \mathbf{d}^{\top} X^{-1} \mathbf{d}\left(-\ln \|x\|_{\mathbf{d}}\right) x}{x^{\top} \mathbf{d}^{\top}\left(-\ln \|x\|_{\mathbf{d}}\right) X^{-1} G_{\mathbf{d}} \mathbf{d}\left(-\ln \|x\|_{\mathbf{d}}\right) x} .
\end{aligned}
$$

The formula (11) implies that the canonical homogeneous norm is a Lyapunov function for the closed-loop system. The feasibility of the algebraic system is proven in [22]. The existence of an appropriate solution for (7) is studied in [28]. It can be shown that for any solution of (7) the matrix $G_{0}-I$ is invertible. Moreover, for a sufficiently small $|\mu|$ we have $G_{\mathbf{d}}=I+\mu G_{0}$ is anti-Hurwitz.

Remark 1: If the matrix $\mathrm{A}$ is nilpotent then $K_{0}=0$. In this case, for $\mu=-1$ any solution $x\left(t, x_{0}\right)$ of the closed loop system (6), (8) with the initial condition $x(x)=x_{0}$ sarisfies

$$
\begin{aligned}
\|u(x)\| & =\left\|Y X^{-1} \mathbf{d}\left(-\ln \|x\|_{\mathrm{d}}\right) x\right\| \\
& \leq\left\|Y X^{-1}\right\| \cdot\left\|\mathrm{d}\left(-\ln \|x\|_{\mathrm{d}}\right) x\right\| \\
& =\left\|Y X^{-1}\right\|<+\infty
\end{aligned}
$$

This means that the homogeneous proportional control solves the stabilization problem without an unbounded "peaking effect" (see [21, Chapter 1] for more details).

\section{MAIn RESUlt: ANALYSIS OF HOMOGENEOUS PI CONTROLLER}

We consider a more general class of perturbations. The disturbance function $g$ is assumed to have the following representation

$$
g(t, x)=g_{1}(t, x)+B p+\int_{0}^{t} B g_{2}(s, x(s)) d s, \quad t \geq 0
$$

where $g_{1}: \mathbb{R}_{+} \times \mathbb{R}^{n} \rightarrow \mathbb{R}^{n}$ and $g_{2}: \mathbb{R}_{+} \times \mathbb{R}^{n} \rightarrow \mathbb{R}^{m}$ are assumed to be locally measurable unknown functions and $p \in \mathbb{R}^{m}$ is a constant unknown vector.

Let us consider the following homogeneous PI controller for linear system (6):

$$
u(x)=K_{0} x+u_{\text {hom }}(x)+\int_{0}^{t} u_{\text {int }}(x(s)) d s
$$

The first step, the proportional part design according to Theorem 2:

$$
u_{\text {hom }}(x)=\|x\|_{\mathbf{d}}^{1+\mu} K \mathbf{d}\left(-\ln \|x\|_{\mathbf{d}}\right) x
$$

where $K_{0}$ and $K$ are given in Theorem 2 .

In the second step, in the integral controller design, we adopt the resemble form of the integral part, which has been studied in [26]:

$$
u_{\text {int }}(x)=\frac{\|x\|_{\mathbf{d}}^{1+2 \mu} \tilde{K} \mathbf{d}\left(-\ln \|x\|_{\mathbf{d}}\right) x}{x^{\top} \mathbf{d}^{\top}\left(-\ln \|x\|_{\mathbf{d}}\right) P G_{\mathbf{d}} \mathbf{d}\left(-\ln \|x\|_{\mathbf{d}}\right) x}
$$

$\tilde{K} \in \mathbb{R}^{m \times n}$ is the gain to be defined.

With the notation

$$
x_{n+1}=p+\int_{0}^{t} u_{\text {int }}(x(s))+g_{2}(s, x(s)) d s
$$

the closed-loop system becomes

$$
\begin{aligned}
\dot{x} & =A x+B\left(K_{0} x+u_{\text {hom }}(x)+x_{n+1}\right)+g_{1}(t, x) \\
\dot{x}_{n+1} & =u_{\text {int }}(x)+g_{2}(t, x) .
\end{aligned}
$$

The system (17) is $\mathbf{d}$-homogeneous of degree $\mu$ for the dilation $\tilde{\mathbf{d}}=\left(\begin{array}{cc}e^{s \mathbf{G}_{d}} & 0 \\ 0 & e^{s(1+\mu)} \mathbf{I}_{m}\end{array}\right)$ when $g_{1}=0$ and $g_{2}=0$.

Theorem 3 (The case of constant perturbation): Let $g_{1}=\mathbf{0}, g_{2}=\mathbf{0}$ and the matrices $G_{\mathbf{d}}, K_{0}, K=Y X^{-1}$, $P=X^{-1}$ be defined as in Theorem 2 for some $\mu \geq-0.5$, $\mu \neq 0$ and some $\rho>0$, then the closed-loop system (6) and (14) is globally uniformly asymptotically stable provided that $\tilde{K}=Q^{-1} \tilde{Y}$, where the matrix $\tilde{Y} \in \mathbb{R}^{m \times n}$ and the symmetric matrix $Q \in \mathbb{R}^{m \times m}$ satisfy the following system of LMIs:

$$
\begin{gathered}
W_{1}=\left(\begin{array}{cc}
\frac{P}{1+\mu} & \tilde{Y}^{\top} \\
\tilde{Y} & Q
\end{array}\right) \succ 0, \quad \gamma>0, \\
\left(\begin{array}{cccc}
-\rho P & \frac{P B+\tilde{Y}^{\top}}{q_{i}}+\frac{(\hat{F}-\rho \tilde{F})^{\top} \tilde{Y}^{\top}}{2} & \tilde{Y}^{\top} & \mathbf{0} \\
* & \frac{\gamma B^{\top} P B}{q_{i}}+\frac{\tilde{Y} B+B^{\top} \tilde{Y}^{\top}}{2} & \mathbf{0} & \tilde{Y} \tilde{F} \\
* & * & -q_{i} Q & \mathbf{0} \\
* & * & * & -\gamma q_{i} P
\end{array}\right) \prec 0,
\end{gathered}
$$


where $R=P G_{\mathbf{d}}+G_{\mathbf{d}}^{\top} P \succ 0, \hat{F}=A_{0}+B K, \tilde{F}=$ $\left((1+\mu) I_{n}-G_{\mathbf{d}}\right), i=1,2$ and $q_{1}=\lambda_{\min }\left(P^{-1 / 2} R P^{-1 / 2}\right)$, $q_{2}=\lambda_{\max }\left(P^{-1 / 2} R P^{-1 / 2}\right)$, then a Lyapunov function of the system (6) and (14) is given by

$$
V(\xi)=\frac{1}{2} \xi^{\top}\left(\begin{array}{cc}
\frac{P}{1+\mu} & \tilde{Y}^{\top} \\
\tilde{Y} & Q
\end{array}\right) \xi .
$$

$$
\begin{array}{r}
\text { where } \xi=\left(\begin{array}{c}
\|x\|_{\mathbf{d}}^{1+\mu} \mathbf{d}\left(-\ln \|x\|_{\mathbf{d}}\right) x \\
x_{n+1}
\end{array}\right) \text { and } \\
\dot{V}(\xi) \leq-\tilde{\rho}\|x\|_{\mathbf{d}}^{\mu} V(\xi), \quad x \neq \mathbf{0},
\end{array}
$$

where $\tilde{\rho}>0$ is a positive number dependent of $\mu, \rho, P, Q$ and $\tilde{Y}$.

Remark 2: $u_{\text {int }}$ is globally bounded and discontinuous at $x=0$ if $\mu=-0.5$. For $\mu>-0.5$, the considered system has the continuous right-hand side.

The main idea of the proof of this theorem is to construct a quadratic (with respect to a special vector dependent of using the canonical homogeneous norm) Lyapunov function. In the previous work [26], it was shown that for $\tilde{Y}=0$ the function (20) is weak Lyapunov function $(\dot{V} \leq 0)$ of the closed-loop system with $\tilde{K}=-Q^{-1} B^{\top} P$, so the stability analysis in this case can be based on the LaSalle invariance principle [13].

For $n=1$ and $\mu=-0.5$ the function (20) coincides with the Lyapunov function introduced in [17] for the supertwisting system, since $\|x\|_{\mathbf{d}}=|x|$ in the scalar case.

For $\mu<0$ the function (20) is a strict Lyapunov function of the closed-loop system due to (21). Hence, it can be utilized for advanced analysis. For instance, we can estimate the settling time based on (21).

Corollary 1 (Finite-time Stability for $\mu<0$ ): Under conditions of Theorem 3 with $\mu<0$, the closed-loop system is finite-time stable and the setting-time admits the estimate $T: \mathbb{R}^{n} \rightarrow \mathbb{R}_{+}$as

$$
T(x) \leq \frac{r^{-\frac{\mu}{2+2 \mu}} V^{-\frac{\mu}{2+2 \mu}}\left(\xi_{0}\right)}{\tilde{\rho} \frac{-\mu}{2+2 \mu}}
$$

where $r=\lambda_{\max }(P) / \tilde{\lambda}$ with

$$
\tilde{\lambda}=\frac{1}{2} \lambda_{\min }\left(\begin{array}{cc}
\frac{P}{1+\mu} & \tilde{Y}^{\top} \\
\tilde{Y} & Q
\end{array}\right)
$$

and

$$
\xi_{0}=\left(\begin{array}{c}
\left\|x_{0}\right\|_{\mathbf{d}}^{1+\mu} \mathbf{d}\left(-\ln \left\|x_{0}\right\|_{\mathbf{d}}\right) x_{0} \\
p
\end{array}\right) .
$$

The settling time is a function of the initial condition $\xi_{0}$. Hence the settling time estimate needs to know the maximum magnitude of the constant perturbation.

In most practice, perturbations are always time-varying. Homogeneous control could further minimize the effect of the disturbance. The homogeneous proportional controller has shown that it can reject a class of vanishing-perturbation. The homogeneous PI controller can reject more classes of non-vanishing perturbations. The case of the nonzero disturbances is studied below.
Corollary 2 (The case non-constant perturbations): Let under conditions of Theorem 3 the following inequalities hold:

$$
\begin{gathered}
\frac{2\left|x^{\top} \mathbf{d}^{\top}\left(-\ln \|x\|_{\mathbf{d}}\right) P \mathbf{d}\left(-\ln \|x\|_{\mathbf{d}}\right) g_{1}(t, x)\right|}{x^{\top} \mathbf{d}^{\top}\left(-\ln \|x\|_{\mathbf{d}}\right) R \mathbf{d}\left(-\ln \|x\|_{\mathbf{d}}\right) x} \leq \gamma_{1}\|x\|^{\mu} \\
\frac{\left|g_{1}^{\top}(t, x) \mathbf{d}^{\top}\left(-\ln \|x\|_{\mathbf{d}}\right) P \mathbf{d}\left(-\ln \|x\|_{\mathbf{d}}\right) g_{1}(t, x)\right|}{4} \leq \tilde{\gamma}_{1}^{2}\|x\|_{\mathbf{d}}^{2 \mu} \\
\left|x^{\top} \mathbf{d}^{\top}\left(-\ln \|x\|_{\mathbf{d}}\right) P B g_{2}(t, x)\right| \leq \gamma_{2}\|x\|_{\mathbf{d}}^{1+2 \mu} \\
q(x) \frac{g_{2}^{\top}(t, x) Q\left(B^{\top} P B\right)^{-1} Q g_{2}(t, x)}{4} \leq \tilde{\gamma}_{2}^{2}\|x\|_{\mathbf{d}}^{2+4 \mu}
\end{gathered}
$$

where the parameters $\gamma_{1}, \tilde{\gamma}_{1}, \gamma_{2}, \tilde{\gamma}_{2} \geq 0$ satisfy the systems of LMIs (18-19) and

$$
\left(\begin{array}{ccccc}
\left(\sum-\rho\right) P, & \frac{P B+\tilde{Y}^{\top}}{q_{i}}+\frac{\left.\left(\hat{F}-\rho \tilde{F} \pm 2 \gamma_{1}\right) \tilde{F}\right)^{\top} \tilde{Y}^{\top}}{2} & \tilde{Y}^{\top} & \mathbf{0} & \mathbf{0} \\
* & \frac{\left(\gamma+\tilde{\gamma}_{1}\right) B^{\top} P B}{q_{i}}+\frac{\tilde{Y} B+B^{\top} \tilde{Y}^{\top}}{2} & 0 & \tilde{Y} \tilde{F} & \mathbf{0} \\
* & * & -q_{i} Q & \mathbf{0} & \mathbf{0} \\
* & * & * & -\gamma q_{i} P & \tilde{Y} \\
* & * & * & * & -\frac{P}{\tilde{\gamma}_{2}}
\end{array}\right) \prec 0
$$

where $\sum=2 \gamma_{1}+\gamma_{2}+\tilde{\gamma}_{1}+\tilde{\gamma}_{2}, i=1,2$. Then the closedloop system (1), (14) is globally uniformly

- globally uniformly finite-time stable for $\mu<0$;

- globally uniformly exponentially stable for $\mu=0$;

- globally uniformly nearly fixed-time stable $\mu>0$.

Based on the strict Lyapunov function, using the property of canonical homogeneous norm, we can embed the perturbation information into new LMIs, which conserve the finite/fixed-time convergence rate.

According to Remark 1 and Remark 2, the terms $u_{h o m}$ and $u_{\text {int }}$ are locally bounded, and the settling time when $\mu<0$ is estimated in Corollary 2, so we can estimate the maximum magnitude of the control signal dependently of the initial condition and the magnitude of the constant perturbation/

Corollary 3 (The estimate of the control magnitude): Under conditions of Theorem 3 (or Corollary 2) one has

$$
\|u(x)\|_{m} \leq\left\|K_{0}\right\| \cdot\|x\|+\|K\| \cdot\|x\|_{\mathbf{d}}^{1+\mu}+\sqrt{\frac{V\left(\xi_{0}\right)}{\tilde{\lambda}}}+\|p\|,
$$

where $\tilde{\lambda}$ and $\xi_{0}$ are defined in Corollary 1, the vector norm in $\mathbb{R}^{m}$ is defined as

$$
\|v\|_{m}=\sqrt{v^{\top} v}
$$

the vector norm in $\mathbb{R}^{n}$ is given by

$$
\|y\|=\sqrt{y^{\top} P y}
$$

and the matrix norm for $Z \in \mathbb{R}^{m \times n}$ is defined as follows

$$
\|Z\|=\sqrt{\lambda_{\max }\left(P^{-1 / 2} Z^{\top} Z P^{-1 / 2}\right)} .
$$

Remark 3: The integral part $x_{n+1}$ is bounded by $\sqrt{\frac{V\left(\xi_{0}\right)}{\tilde{\lambda}}}+\|p\|$, when the initial state and the constant perturbation are bounded, $x_{n+1}$ is bounded.

Compared to homogeneous proportional control, the admissible value of the homogeneity degree of the homogeneous PI algorithm is restricted to $\mu \geq-0.5$, since for $\mu<-0.5$ the term $u_{\text {int }}$ becomes unbounded at $x=0$. 


\section{EXAMPLE}

A simulation on a twin rotor system is implemented. The twin rotor system (TRMS 33-220 platform) model can be represented in the following form [28]:

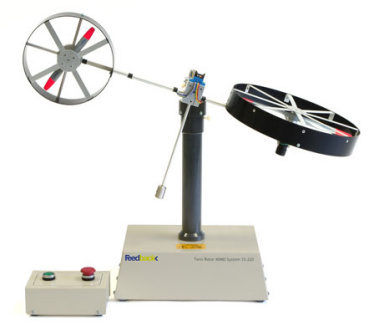

Fig. 1. TRMS 33-220 twin rotor MIMO system

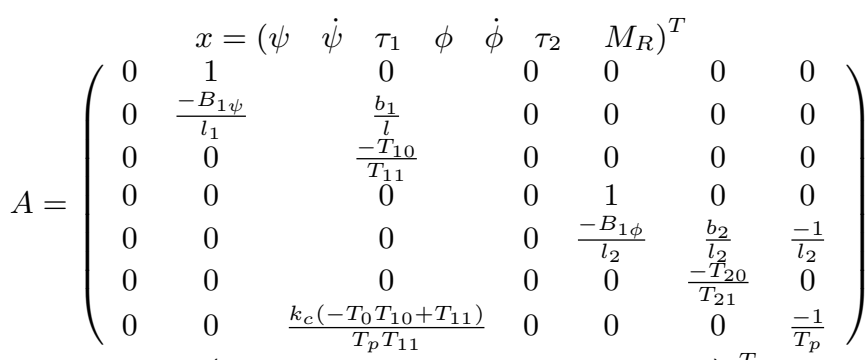

$$
\begin{aligned}
& B=\left(\begin{array}{ccccccc}
0 & 0 & \frac{k_{1}}{T_{11}} & 0 & 0 & 0 & \frac{k_{c} T_{0} k_{1}}{T_{p} T_{11}} \\
0 & 0 & 0 & 0 & 0 & \frac{k_{2}}{T_{21}} & 0
\end{array}\right)^{T}
\end{aligned}
$$

where $\psi$ is the pitch angle, $\phi$ is the yaw angle, $\tau_{1}, \tau_{1}$ are the vertical and horizontal motor moments, $M_{R}$ is the cross reaction momentum. With $\mu=-0.02, \rho=1$, Then, by solving the LMIs (9) and (18-19) we obtain:

$$
\begin{aligned}
& K_{0}=\left(\begin{array}{cc}
0 & 0 \\
0.0012 & -0.0063 \\
0.0807 & 2.6835 \\
0 & 0 \\
0 & -6.9444 \\
0 & 7.500 \\
-4.0476 & -56.5131
\end{array}\right)^{\top} \\
& K=10^{3}\left(\begin{array}{cc}
-0.1591 & -0.0067 \\
0.0798 & 0.0134 \\
-0.9316 & -0.0401 \\
-0.0080 & -0.0053 \\
-0.0150 & -0.0100 \\
0.0034 & 0.0057 \\
-2.6709 & -0.1779
\end{array}\right) \\
& G_{d}=10^{-2}\left(\begin{array}{ccccccc}
106 & -4.86 & 30.8 & -0 & 0 & 0 & 88 \\
0 & 104.43 & -15.40 & 0 & 0 & 0 & -44 \\
& 0.13 & 104.67 & 0 & 0 & 0 & 13.33 \\
-0.26 & 0.64 & -4.03 & 104 & 0 & 0 & -11.51 \\
0 & -0.32 & 2.02 & 0 & 102 & 0 & 5.76 \\
0 & -0.59 & -10.02 & 0 & 2.22 & 100 & -28.63 \\
0 & -0.02 & -1.08 & 0 & 0 & 0 & 96.9
\end{array}\right) \\
& P=10^{3}\left(\begin{array}{ccccccc}
0.65 & -0.50 & 4.55 & -0.02 & 0 & -0.01 & 13 \\
-0.5 & 0.6 & -4.24 & -0.01 & 0 & 0 & -12.12 \\
4.55 & -4.24 & 34.96 & -0.14 & 0 & -0.07 & 99.82 \\
-0.02 & -0.01 & -0.14 & 0.01 & 0 & 0 & -0.39 \\
0 & 0 & 0 & 0 & 0 & 0 & 0.01 \\
-0.01 & 0 & -0.07 & 0 & 0 & 0 & -0.19 \\
13 & -12.12 & 99.82 & -0.39 & 0.01 & -0.19 & 285
\end{array}\right)
\end{aligned}
$$

$$
\begin{gathered}
\tilde{K}=\left(\begin{array}{cc}
-159.1 & -6.7 \\
-79.8 & 13.4 \\
-931.6 & 40.1 \\
-8 & -5.3 \\
-15 & -10 \\
3.4 & 5.7 \\
-2670.9 & -177.9
\end{array}\right)^{\top}, \tilde{Y}=\left(\begin{array}{cc}
0.1024 & -0.3513 \\
-0.9187 & 0.4149 \\
0.9008 & 10.1486 \\
-0.3336 & 0.0903 \\
-8.6533 & -3.8911 \\
0 & 4.3103 \\
-0.3688 & -3.5183
\end{array}\right)^{\top} \\
Q=\left(\begin{array}{cc}
0.1496 & -0.0464 \\
-0.0464 & 0.3041
\end{array}\right), \gamma=0.0094
\end{gathered}
$$

The initial state is $[0.5,0,0,0.5,0,0,0]^{\top}$, the constant pertubation $p=1$. Simulation results are presented in Fig. 2 and Fig. 3. From the result, the system has a static error under the homogeneous $\mathrm{P}$ controller, but the system with the homogeneous PI controller can well reject the constant perturbation. Moreover, the system has a non-asymptotic convergence rate.
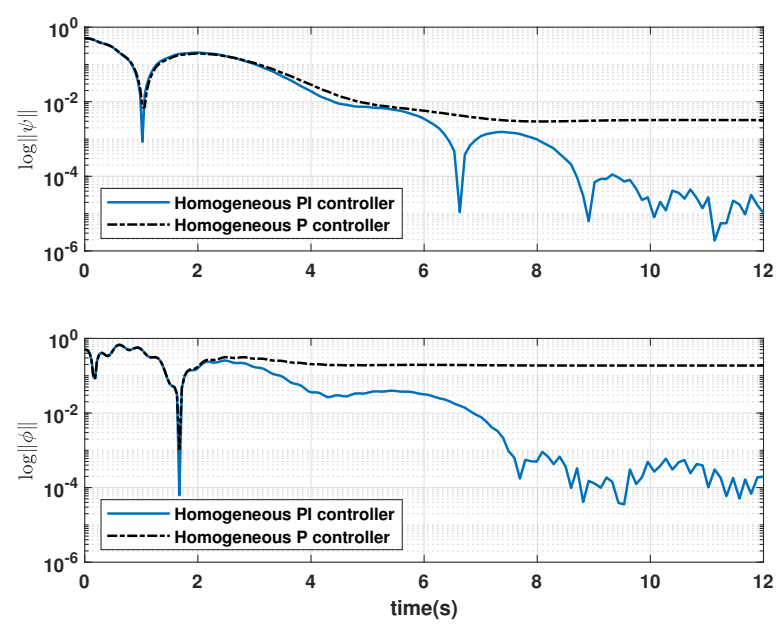

Fig. 2. Angular position under homogeneous PI and P controller
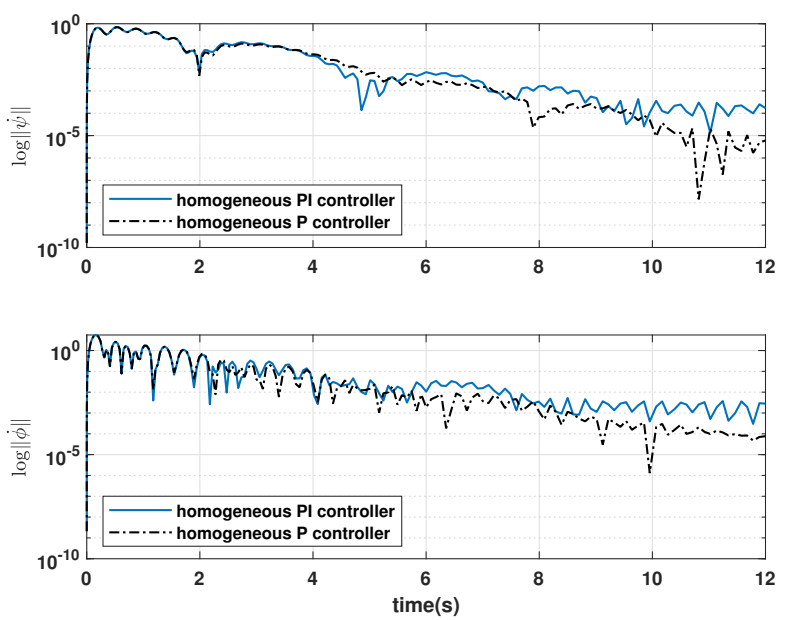

Fig. 3. Angular velocity under homogeneous PI and P controller 


\section{CONCLUSION}

A strictly Lyapunov function for an analysis of the generalized homogeneous PI controller with negative homogeneity degree is designed. A class of perturbations to be rejected by the mentioned controller is characterized. Moreover, the settling time and the magnitude of the control signal are estimated dependently of the initial condition and the magnitude of the perturbation. A further study about the case of a positive homogeneity degree will need to be done to obtain the nearly fixed time stability property.

\section{REFERENCES}

[1] A. Bacciotti and L. Rosier. Lyapunov Functions and Stability in Control Theory. Springer, 2001.

[2] E. Bernuau, D. Efimov, W. Perruquetti, and A. Polyakov. On homogeneity and its application in sliding mode control. Journal of The Franklin Institute, 351(4):1866-1901, 2014.

[3] S. P. Bhat and D. S. Bernstein. Geometric homogeneity with applications to finite-time stability. Mathematics of Control, Signals and Systems, 17:101-127, 2005.

[4] V. Fischer and M. Ruzhansky. Quantization on Nilpotent Lie Groups. Springer, 2016.

[5] L. Grüne. Homogeneous state feedback stabilization of homogeneous systems. SIAM Journal of Control and Optimization, 38(4):1288$1308,2000$.

[6] L.S. Husch. Topological Characterization of The Dilation and The Translation in Frechet Spaces. Mathematical Annals, 190:1-5, 1970.

[7] Z. Jiang and I Marcels. Robust nonlinear integral control. IEEE Transactions on Automatic Control, 46(8):1336-1342, 2001.

[8] M. Kawski. Homogeneous stabilizing feedback laws. Control Theory and Advanced Technology, 6(4):497-516, 1990.

[9] M. Kawski. Families of dilations and asymptotic stability. Analysis of Controlled Dynamical Systems, pages 285-294, 1991.

[10] V. V. Khomenuk. On systems of ordinary differential equations with generalized homogenous right-hand sides. Izvestia vuzov. Mathematica (in Russian), 3(22):157-164, 1961.

[11] G. C Konstantopoulos, Q-C Zhong, B. Ren, and M. Krstic. Bounded integral control of input-to-state practically stable nonlinear systems to guarantee closed-loop stability. IEEE Transactions on Automatic Control, 61(12):4196-4202, 2016.

[12] N. J. Krikelis. State feedback integral control with 'intelligent'integrators. International Journal of Control, 32(3):465-473, 1980.

[13] J. La Salle and S. Lefschetz. tability by Liapunov's Direct Method with Applications. Elsevier Science, 1961.

[14] A. Levant. Sliding order and sliding accuracy in sliding mode control. International Journal of Control, 58(6):1247-1263, 1993.

[15] A. Levant. Homogeneity approach to high-order sliding mode design. Automatica, 41(5):823-830, 2005.

[16] A. Mercado-Uribe, J. A Moreno, A. Polyakov, and D. Efimov. Multiple-input multiple-output homogeneous integral control design using the implicit lyapunov function approach. International Journal of Robust and Nonlinear Control, 31(9):3417-3438, 2021.

[17] J. Moreno and M. Osorio. Strict Lyapunov functions for the super-twisting algorithm. IEEE Transactions on Automatic Control, 57:1035-1040, 2012.

[18] Y. Orlov. Finite time stability and robust control synthesis of uncertain switched systems. SIAM Journal of Control and Optimization, 43(4):1253-1271, 2005.

[19] M. Pachter, J. D'AZZO, and M. Veth. Proportional and integral control of nonlinear systems. International Journal of Control, 64(4):679-692, 1996.

[20] A. Polyakov. Sliding mode control design using canonical homogeneous norm. International Journal of Robust and Nonlinear Control, 29(3):682-701, 2018.

[21] A. Polyakov. Generalized Homogeneity in Systems and Control. Springer, 2020.

[22] A. Polyakov, D. Efimov, and W. Perruquetti. Robust stabilization of MIMO systems in finite/fixed time. International Journal of Robust and Nonlinear Control, 26(1):69-90, 2016.
[23] L. Rosier. Homogeneous Lyapunov function for homogeneous continuous vector field. Systems \& Control Letters, 19:467-473, 1992.

[24] M. Rubagotti, A. Estrada, F. Castaños, A. Ferrara, and L. Fridman Integral sliding mode control for nonlinear systems with matched and unmatched perturbations. IEEE Transactions on Automatic Control, 56(11):2699-2704, 2011.

[25] V. I. Utkin. Sliding Modes in Control Optimization. Springer-Verlag, Berlin, 1992.

[26] S. Wang, A. Polyakov, and G. Zheng. Generalized homogenization of linear controllers: Theory and experiment. International Journal of Robust and Nonlinear Control, 31(9):3455-3479, 2021.

[27] S. Wang, A. Polyakov, and G. Zheng. Upgrade of pid controller using generalized homogeneity, Patent: FR2004684, 2020.

[28] K. Zimenko, A. Polyakov, D. Efimov, and W. Perruquetti. Robust feedback stabilization of linear mimo systems using generalized homogenization. IEEE Transactions on Automatic Control, 65(12):54295436, 2020.

[29] V.I. Zubov. On systems of ordinary differential equations with generalized homogenous right-hand sides. Izvestia vuzov. Mathematica (in Russian), 1:80-88, 1958. 